\title{
Note de cadrage sur la linguistique textuelle
}

\author{
Jean-Michel ADAM \\ (Université de Lausanne)
}

Quand on dit qu'un énoncé fait sens, il fait d'abord texte. Antoine Culioli (2003:147-148)

Dans le dernier chapitre de Linguistique (2005 [1995]), Olivier Soutet souligne le caractère paradoxal de la linguistique textuelle ${ }^{\mathrm{i}}$ :

\begin{abstract}
La linguistique textuelle est [...] une discipline quelque peu paradoxale. Evaluée à l'aune de ce qu'il est convenu d'appeler la linguistique moderne - celle qui nous conduit du comparatisme historiciste du début du XIX $^{\mathrm{e}}$ siècle au poststructuralisme du dernier tiers du XX $X^{\mathrm{e}}$ siècle -, elle paraît toute jeune et en quête de légitimité ; replacée dans la longue durée des savoirs et des techniques - philologie, littéraire et judiciaire - qui ont pour objet, sinon le texte en général, du moins certains types de textes, elle semble n'en être que le prolongement ou l'élargissement. (1995:324)
\end{abstract}

La linguistique textuelle a effectivement quelque chose à voir avec la rhétorique qui prenait en charge la dispositio du texte oratoire, avec la stylistique qui a(vait) pour objet la texture (elocutio) des textes littéraires, avec la poétique qui a(vait) pour double objet la structure des textes et la question des genres littéraires, avec l'herméneutique philologique, ses savoirs et ses techniques d'établissement du texte et de son analyse, sans oublier la traduction qui trouve sa pleine réalisation textuelle et discursive dans la Poétique de la traduction d'Henri Meschonnic (1999). Le réexamen de ces disciplines est, à l'aube du $\mathrm{XXI}^{\mathrm{e}}$ siècle, un des chantiers interdisciplinaires les plus stimulants (Adam 2005), à condition toutefois de ne pas tomber dans une illusion continuiste de l'histoire des sciences et de poursuivre le combat nécessaire contre les présupposés métaphysiques, l'essentialisme et l'oubli de l'histoire des sciences et de l'épistémologie.

Au regard de la linguistique moderne, la linguistique textuelle est effectivement une discipline encore jeune dont la légitimité est toutefois de plus en plus reconnue. Apparue dans les années 1950 en Allemagne, elle s'est développée dans le cadre du second Cercle de Prague (Mathesius 1929, Danes 1978 et Firbas 1964) et, en Angleterre, chez Halliday et Hasan (1976) qui sont les premiers à ne pas grammaticaliser le transphrastique en considérant le texte comme une grande phrase ou comme une simple suite de phrases :

\footnotetext{
Un texte [...] n'est pas un simple enchaînement de phrases [string of sentences]. En d'autres termes, il ne s'agit pas d'une grande unité grammaticale, de quelque chose de même nature qu'une phrase mais qui en différerait par la taille-une sorte de superphrase. Un texte ne doit pas du tout être vu comme une unité grammaticale, mais comme une unité d'une autre espèce : une unité sémantique. Son unité est une unité de sens en contexte, une texture qui exprime le fait que, formant un tout [as a whole], il est lié à l'environnement dans lequel il se trouve placé. (1976: 293; notre traduction)
}

Dans ses derniers travaux, Eugenio Coseriu, qui a été un des premiers à employer le terme «linguistique textuelle », propose de distinguer la " grammaire transphrastique » de la «linguistique textuelle » (1994). Si la première peut être considérée comme une extension de la linguistique classique, la linguistique textuelle est, en revanche, une théorie de la production co(n)textuelle de sens qu'il est nécessaire de fonder sur l'analyse de textes concrets. C'est cette démarche que je nomme, pour ma part, analyse textuelle des discours. 
Je montre ailleurs que, selon moi, la linguistique textuelle est aussi continuatrice de la définition du discours que se donnent des linguistes fondateurs de la linguistique moderne comme Ferdinand de Saussure, Gustave Guillaume et surtout Emile Benveniste (Adam 2001b \& 2008 : 15-24). Benveniste se sépare de Saussure et se rapproche de Guillaume en instaurant dans la langue "une division fondamentale, toute différente de celle que Saussure a tentée entre langue et parole » (1974: 224). Comme Guillaume faisait de la phrase «l'unité d'effet du langage » (Leçon du 19-11-1948, série C), Benveniste la considère comme l'unité de la communication humaine et, la repoussant au-delà du dernier niveau de l'échelle des combinaisons linguistiques codées, il ajoute: "Avec la phrase une limite est franchie, nous entrons dans un nouveau domaine. [...] Elle se distingue foncièrement des autres entités linguistiques » (1966: 128).

Dans son modèle des niveaux de l'analyse linguistique, la limite inférieure du système est constituée par les «mérismes», traits distinctifs de phonèmes, qui ne comportent aucun constituant de nature linguistique et ne se définissent que par leur nature de constituant d'une unité linguistique de rang supérieur. La phrase, qui ne se définit que par ses constituants, ne peut en revanche intégrer aucune unité de rang plus élevé de complexité. Entre ces deux niveaux, les signes, mots ou morphèmes «à la fois contiennent des constituants et fonctionnent comme intégrants » $(1966: 125)$. Tout cela repose sur une définition de la forme et du sens que se donne Benveniste :

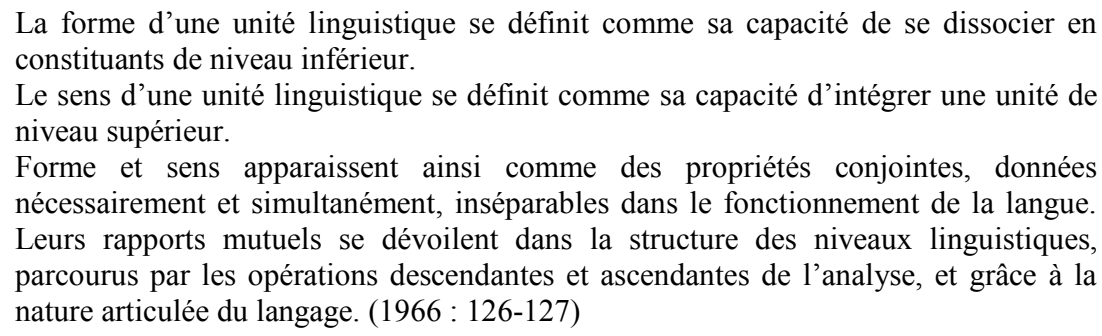

En faisant de la proposition l'unité de dernier rang intégratif, Benveniste pose les limites de la linguistique du système :

Du signe à la phrase il n’y a pas transition, ni par syntagmation ni autrement. Un hiatus les sépare. Il faut alors admettre que la langue comporte deux domaines distincts, dont chacun demande son propre appareil conceptuel. Pour celui que nous appelons sémiotique, la théorie saussurienne du signe linguistique servira de base à la recherche. Le domaine sémantique, par contre, doit être reconnu comme séparé. Il aura besoin d'un appareil nouveau de concepts et de définitions. (1974:65)

Pour Benveniste, comme pour Guillaume et Saussure, la phrase est déjà une unité d'un autre ordre : «La phrase appartient bien au discours. C'est même par là qu'on peut la définir: la phrase est l'unité du discours. [...] La phrase est une unité, en ce qu'elle est un segment de discours » (1966: 130). Pour Benveniste :

[...] Avec la phrase on quitte le domaine de la langue comme système de signes, et l'on entre dans un autre univers, celui de la langue comme instrument de communication, dont l'expression est le discours.

Ce sont là vraiment deux univers différents, bien qu'ils embrassent la même réalité, et ils donnent lieu à deux linguistiques différentes, bien que leurs chemins se croisent à tout moment. (1966: 129-130)

En esquissant avec «L'appareil formel de l'énonciation» (1974 : 79-88) les contours de la linguistique de l'énonciation, Benveniste ne se contente pas d'ouvrir l'analyse intralinguistique à la sémantique de l'énonciation. En effet, si la théorie de l'énonciation a pour objet la production d'énoncés et non le « texte de l'énoncé » (1974:80), c'est qu'une autre branche de la linguistique est appelée à prendre ce dernier en charge. C'est ce qu'il explique à la fin de son dernier article publié (Semiotica 1969) : 


\begin{abstract}
En conclusion, il faut dépasser la notion saussurienne du signe comme principe unique, dont dépendraient à la fois la structure et le fonctionnement de la langue. Ce dépassement se fera par deux voies :

- dans l'analyse intralinguistique, par l'ouverture d'une nouvelle dimension de signifiance, celle du discours, que nous appelons sémantique, désormais distincte de celle qui est liée au signe, et qui sera sémiotique ;

- dans l'analyse translinguistique des textes, des œuvres par l'élaboration d'une métasémantique qui se construira sur la sémantique de l'énonciation.

Ce sera une sémiologie de "deuxième génération", dont les instruments et la méthode pourront aussi concourir au développement des autres branches de la sémiologie générale. (1974:66)
\end{abstract}

Benveniste décompose programmatiquement le champ général de la linguistique en deux domaines (analyse intralinguistique et analyse translinguistique) entre lesquels la linguistique de l'énonciation occupe une place centrale. Prenant appui sur cette dernière, la linguistique du discours est, d'une part, une «translinguistique des textes » et, d'autre part, une «translinguistique des œuvres », c'est-à-dire des productions littéraires. Je considère ces lignes programmatique comme un appel à réunir la linguistique de l'énonciation et la linguistique textuelle au sein de la linguistique du discours.

Roland Barthes est, avec Henri Meschonnic et Julia Kristeva, un des rares a avoir compris l'importance du programme de Benveniste. Il y fait allusion dès le numéro 8 de Communications : « Le discours a ses unités, ses règles, sa "grammaire" : au-delà de la phrase et quoique composé uniquement de phrases, le discours doit être naturellement l'objet d'une seconde linguistique » (1966: 3). Cette "seconde linguistique », Barthes la nommera jusqu'en 1970 «linguistique du discours ou translinguistique (le terme méta-linguistique, préférable, étant déjà pris dans un sens différent)» (2002:611) ${ }^{\mathrm{ii}}$, prolongeant jusque dans le choix des mots le programme de travail de Benveniste :

La translinguistique ne peut [...] se constituer si elle n'établit pas, pour chacun de ses objets, les niveaux d'intégration du discours, depuis la phrase, qui est le dernier niveau d'intégration linguistique et le premier niveau d'intégration translinguistique, jusqu'au moment où le discours s'articule sur la praxis sociale. (Barthes 2002 [1970] : 970)

Nous reviendrons en conclusion sur cette articulation du discours «sur la praxis sociale » et sur l'identification du genre comme "premier niveau sûr » d'intégration de la « suite hiérarchisée des autres niveaux » (2002 [1970] : 971). Il y a certainement ici un excès dans l'application de la théorie de niveaux de l'analyse linguistique.

Certes si, restant strictement dans le «sémiotique » et la linguistique des faits de langue, on renonce à décrire l'intégration de la phrase dans un texte, on renonce à toute science «translinguistique des textes et des œuvres » et, en fait, à toute linguistique du discours. Mais cette intégration obéit-elle aux mêmes lois ? Si on repart du dispositif de Benveniste et du fait que « le sens d'une unité linguistique se définit comme sa capacité d'intégrer une unité de niveau supérieur » (1966:127), la question de l'intégration des phrases dans un texte se pose en fait tout autrement. La question devient: existe-t-il des unités segmentables en unités de niveau inférieur entre le niveau du texte et celui de la phrase qui permettrait de les définir comme des formes linguistiques?

Pour certains linguistes, comme Marc Wilmet, «le texte se présente comme une extrapolation de la phrase » et il ajoute cette mise en garde : «Les linguistes aventurés aux confins de leur domaine n'auront jamais à abandonner les outils qu'ils se sont patiemment forgés » (1997: 582). C'est également la position défendue par Martin Riegel. Définissant simplement le texte comme un «ensemble organisé de phrases », il propose de compléter les « outils d'une bonne grammaire phrastique » par une simple prise en compte des «conditionnements proprement textuels des phrases lorsqu'elles sont mises en séquence » (2006 : 53). Dans cette perspective, l'intégration transphrastique se poursuit jusqu'au niveau textuel sans solution de continuité.

A cela, on peut opposer cette remarque d'Olivier Soutet : « Dans le cas particulier du texte, le rapport du tout à la partie ne relève pas du même type de prévisibilité que celui qui existe entre chacune des unités 
subphrastiques et leurs constituants immédiats » $(1995: 325)$. Les solidarités syntaxiques entre unités de la langue n'ont, en effet, qu'une portée très limitée. Dès que l'on passe le seuil du syntagme et du noyau de la phrase de base pour entrer dans les domaines de la phrase périodique et du transphrastique, d'autres systèmes de connexions apparaissent, qui ne reposent pas sur des critères morpho-syntaxiques, mais sur des marques et des instructions relationnelles ascendantes et descendantes de portée plus ou moins lointaine. Ces marques de connexion ont « pour fonction conventionnelle de signaler au destinataire que telle ou telle unité doit être comprise comme entretenant telle relation avec telle ou telle autre » (Charolles 1993 : 311). La tâche de la linguistique textuelle est de définir la portée et de catégoriser ces marques d'ouverture ou de fermeture des segments textuels plus ou moins longs, qui ne recoupent que partiellement les catégories morpho-syntaxiques définies dans le cadre de la linguistique de la langue. La cohésion et la cohérence du texte n'étant pas les résultantes de faits de grammaticalité (Combettes 1992 : 113), les domaines textuel et morpho-syntaxique sont différents et assez largement indépendants. Cette "distorsion », ce décalage entre les catégories de la grammaire et celles de la linguistique du texte ne doivent pas étonner. Comme l'a écrit Combettes, les concepts utilisés en grammaire phrastique :
[...] possèdent leur propre utilité, dans leur ordre, mais ne peuvent être " réutilisés » tels quels, dans une problématique qui s'attache à un autre domaine que le leur. [...]. Même si des relations, des interactions, peuvent être observées entre les deux domaines (phrase et texte), des catégories fondées sur des caractéristiques purement grammaticales ne peuvent être considérées comme des notions fondamentales pertinentes en ce qui concerne la cohérence du texte. (1992:113-114)

Combettes (1992) insiste fort justement sur le fait que :

[...] l'opposition phrase/texte ne fait pas le tri entre des phénomènes linguistiques qui relèveraient de la phrase et ceux qui relèveraient du texte, mais elle s'attache à distinguer des propriétés diverses - les unes phrastiques, les autres textuelles - d'une même structure de langue. (1993: 47)

Ces marques instructionnelles n'entrant pas dans des schémas grammaticalement préétablis, le texte apparaît comme une «entité structuralement ouverte » (Charolles $1993: 311$ ).

Dans des travaux plus récents, Michele Prandi se propose de dépasser la «perspective phraséocentrique traditionnelle » $(2007: 75)$ en passant «de la dimension phrastique, régie par la grammaire, à une dimension textuelle, régie par la cohérence des concepts supportée par des moyens cohésifs appropriés » (ibid.). Il parle de " grammaire de règles » pour les « régions de la grammaire qui fonctionnent comme des systèmes de structures et de règles de construction non négociables » $(2007: 71)$ et il parle, en revanche, de "grammaire d'options" pour ces cas où un éventail d'options fonctionnellement équivalentes, une sorte de répertoire d'options, sont au service des projets communicatifs du sujet parlant. Ce franchissement de la frontière du noyau de la phrase simple n'est pas une simple extension transphrastique des limites de la linguistique, il s'agit d'une rupture par rapport à une tradition qui s'obstine à privilégier «les formes par rapport aux contenus, les règles par rapport aux options, la dimension phrastique par rapport à la dimension textuelle » (2007: 75).

Au-delà du tissage grammatical phrastique et trans-phrastique (anaphores et co-référence, connecteurs), c'est par les « réseau de déterminations » (Weinrich $1973: 174$ ) que le texte se donne comme un système organisé de « relations d'interdépendance ». La dimension réticulaire de la distribution des formes verbotemporelles tout au long de la surface textuelle a été très tôt perçue par Harald Weinrich :

Les formes temporelles viennent d'abord à nous - et nous reviennent - à travers des textes. C'est là qu'elles dessinent, avec d'autres signes, et aussi avec d'autres temps, un complexe de déterminations, un réseau de valeurs textuelles [...]. (1973:13)

Les diverses formes verbo-temporelles s'associent en formant des plans d'énonciation dont les enchaînements (continuité et ruptures) dépassent largement les frontières phrastiques dans lesquelles certaines descriptions linguistiques les cantonnent au lieu de rendre compte des groupements de sousensembles locaux. 
Sans remettre en cause le caractère structurant de la dimension linéaire des textes, nous devons «éviter que cette classe de propriétés ne "bénéficie" d'un privilège outrancier, voire exclusif » (Viprey 2006: 74). Tout texte se présente comme une combinaison du linéaire (contraint en grande partie par la linéarité orientée des énoncés et des textes) et de deux modes non-linéaires de construction de sens : la perception d'un tout de sens qui fait l'unité du texte (dimension configurationnelle) et la perception de réseaux complexes de sens (dimension réticulaire). Dominique Legallois souligne fort justement le fait que « L'organisation réticulaire du texte est en parfaite congruence avec l'étymologie du mot texte. C'est bien un tissu de phrases enchevêtrées, une trame, une texture, toute une construction et une conception d'un objet complexe que met en évidence l'analyse de la répétition lexicale dans les discours » (2006: 70). L'identification de répétitions de lexèmes ou de collocations de lexèmes est un facteur cohésif important. La cooccurrence est même un facteur déterminant de la textualité. Passer du lexique (en langue) au vocabulaire propre à un texte (sa structuration en vocables) demande un regard et des méthodes (Viprey 2006 a \& b) affranchies de la linéarité : ce qu'autorise le traitement automatique des données textuelles.

Depuis leur émergence, dans les années 1950, l'analyse de discours et la linguistique textuelle se sont développées de façon autonome. Elles ne se sont guère croisées que dans les travaux de Denis Slakta (1977). C'est sur de nouvelles bases que l'on peut aujourd'hui mettre en relation une linguistique textuelle débarrassée des illusions de la grammaire de texte et des typologies de texte et une analyse de discours renouvelée (Maingueneau 1991 \& 1995).

Le schéma suivant (Adam 2008: 25) tente de mettre en évidence le double jeu des déterminations textuelles «ascendantes » (de droite à gauche) qui régissent les agencements de propositions au sein du système que constitue l'unité texte et des régulations «descendantes» (de gauche à droite) que les situations d'interaction dans des lieux sociaux, des langues et des genres donnés imposent aux énoncés. Sous l'impact des besoins d'expression et des nécessités de l'interaction, les énoncés prennent des formes variationnelles potentiellement infinies, mais les genres de discours et les choix de langues interviennent comme facteurs déterminants de régulation.

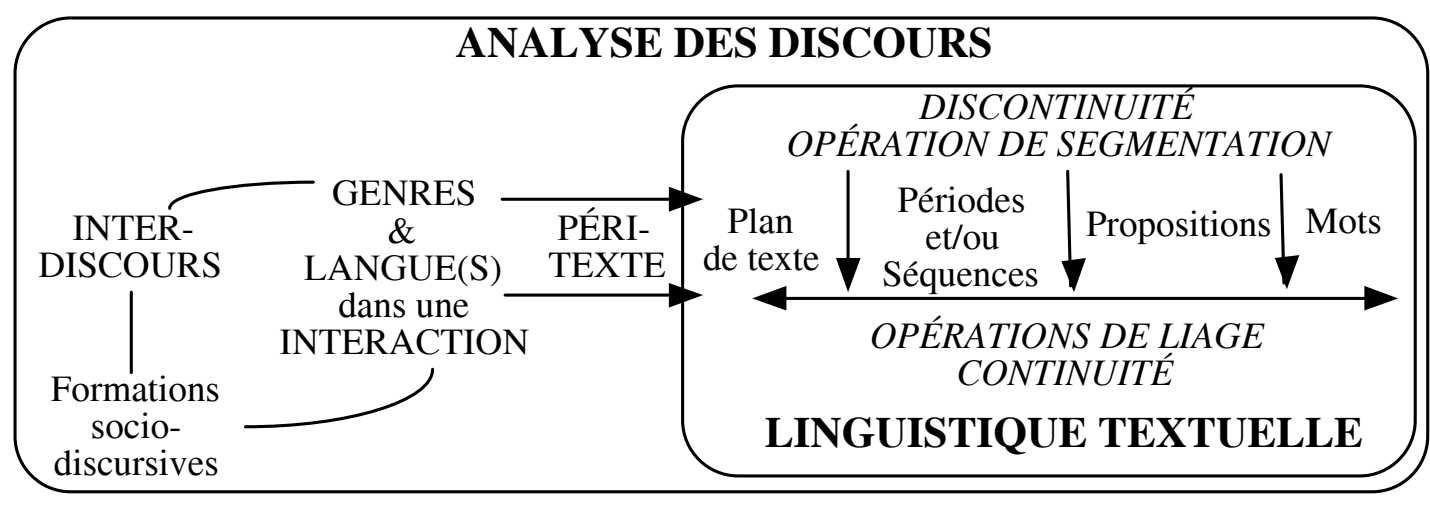

L'établissement d'un lien entre les genres et les formations socio-discursives est une des avancées récentes de l'analyse de discours (Maingueneau 2004), toutefois, dès 1978, Tzvetan Todorov mettait déjà en avant le fait que «N'importe quelle propriété verbale, facultative au niveau de la langue, peut être rendue obligatoire dans le discours. [...]. Certaines règles discursives ont ceci de paradoxal qu'elles consistent à lever une règle de la langue »(1978: 23-24). Dans Les genres de discours, Todorov inscrivait très clairement les systèmes de genres dans l'espace socio-discursif d'un lieu social donné. Pour qu'un sens soit prêté à un texte, il faut qu'il soit en quelque sorte projeté sur «l'arrière-plan d'un schème discursif préexistant» (Stierle 1977: 427), qu'il trouve une place "dans les institutions de l'action symbolique, qui ont pour condition et conditionnent en même temps une culture donnée » (1977: 426). C'est dans les genres de discours que je localise cette "stabilisation publique et normative » qui opère dans le cadre du système de genres de chaque formation discursive. 
Dans L'Archéologie du savoir (1969), Michel Foucault montrait qu'une unité linguistique (phrase ou proposition) ne devient unité de discours (énoncé) que si on relie cet énoncé à d'autres, si on le met en rapport avec un « champ adjacent », un « espace collatéral »: «Un énoncé a toujours des marges peuplées d'autres énoncés » (1969: 128). Foucault mettait ainsi l'accent sur le fait que la langue ne suffit pas à produire à elle seule des énoncés :

Ce ne sont ni la même syntaxe, ni le même vocabulaire qui sont mis en œuvre dans un texte écrit et dans une conversation, sur un journal et dans un livre, dans une lettre et sur une affiche; bien plus, il y a des suites de mots qui forment des phrases bien individualisées et parfaitement acceptables, si elles figurent dans les gros titres d'un journal, et qui pourtant, au fil d'une conversation, ne pourraient jamais valoir comme phrase ayant un sens. (1969: 133)

Lausanne, avril 2008

\section{Références bibliographiques}

ADAM Jean-Michel 1990 : Éléments de linguistique textuelle, Bruxelles-Liège, Mardaga.

- 1999 : Linguistique textuelle : des genres de discours aux textes, Paris, Nathan.

- 2001a: «Barthes en 1970: de la translinguistique à la déconstruction », in A. Boissinot et alii (éd.), Littérature et sciences humaines, Paris, Les Belles-Lettres-CRTH de l'université de Cergy-Pontoise, 125-148.

- 2001b: «Discours et interdisciplinarité. Benveniste lecteur de Saussure », Cahiers Ferdinand de Saussure 54, Genève, Droz, 241-258.

- 2005 : «Les sciences de l'établissement du texte et la question de la variation », in J.-M. Adam \& U. Heidmann (éds.), Sciences du texte et analyse de discours, Genève, Slatkine, 69-96.

- 2006 : «Texte, contexte et discours en question », Pratiques 129-130, Metz, 21-34.

- 2008 [2005] : La Linguistique textuelle. Introduction à l'analyse textuelle des discours, Paris, A. Colin.

Adam Jean-Michel, Grize Jean-Blaise, Ali Bouacha Magid 2004: Texte et discours : catégories pour l'analyse, Dijon, Editions Universitaires de Dijon.

Barthes Roland 2002 (1966) : «Introduction à l'analyse structurale des récits », CEuvres complètes II, Paris, Seuil, $828-865$

- 2002 (1970) : «La linguistique du discours », CEuvres complètes III, Paris, Seuil, 611-616.

Benveniste Emile 1966 : Problèmes de linguistique générale I, Paris, Gallimard.

- 1974 : Problèmes de linguistique générale II, Paris, Gallimard.

Charolles Michel 1993 : «Les plans d'organisation du discours et leurs interactions », in Parcours linguistiques de discours spécialisés, Sophie Moirand et alii (éd.), Peter Lang, Berne, 301-314.

Combettes Bernard 1992 : «Questions de méthode et de contenu en linguistique du texte », Études de linguistique appliquée 87, Paris, Didier, 107-116.

— 2006 : «Textualité et systèmes linguistiques », in Cohérence et discours, Frédéric Calas (dir.), Paris, Presses de l’Université Paris Sorbonne, 39-52.

Coseriu Eugenio 1994 (1980) : Textlinguistik. Eine Einführung, Tübingen-Basel, Francke.

Culioli Antoine 1984 : Préface de La Langue au ras du texte, F. Atlani et alii., Presses universitaires de Lille, 9-12.

- 2003 : «Un linguiste face aux textes saussuriens », Saussure, Cahiers de L’Herne, Paris, 137-149.

Danes Frantisek1978: «De la structure sémantique et thématique du message », Linguistique et sémiologie 5, Presses universitaires de Lyon, 177-200.

Firbas Jan 1964 : «On defining the theme in functional sentence analysis », Travaux linguistiques de Prague, vol. 1, 267-280.

Foucault Michel 1969 : L’Archéologie du savoir, Paris, Gallimard. 
Gardes Tamine Joëlle 2004 : Pour une grammaire de l'écrit, Paris, Belin.

Guillaume Gustave 1985 : Leçons de linguistique de Gustave Guillaume 1945-1946.C, édités par Roch Valin, Walter Hirtle \& André Joly, P. U. Laval-Québec \& P.U.Lille.

— 1973 : Leçons de linguistique de Gustave Guillaume 1948-1949, édités par Roch Valin, Québec, P. U. Laval.

Halliday Michael A. K., Hasan Ruqaiya 1976 : Cohesion in English, Longman, London-New York ; 15e éd. 1997.

Jaubert Anna 2006 : «Les ordres du discours en perspective : cohérence et pertinence », in Cohérence et discours, Frédéric Calas (dir.), Paris, Presses de l’Université Paris Sorbonne, 15-21.

Legallois Dominique 2006 : «Présentation générale. Le texte et le problème de son et ses unités : proposition pour une déclinaisons » \& « Des phrases entre elles à l'unité réticulaire du texte », Langages $n^{\circ} 163$, Paris, Larousse, 3-9 \& 56-70.

Maingueneau Dominique 1991a : L’Analyse du discours, Paris, Hachette.

- 1991b : L'énonciation en linguistique française, Paris, Hachette.

- 1995 (éd.) : «Les analyses du discours en France », Langages 117, Paris, Larousse.

- 2004 : «Retour sur une catégorie : le genre », in J.-M. Adam et al. Texte et discours : catégories pour l'analyse, Dijon, Editions Universitaires de Dijon, 107-118.

Mathesius Vilém (1929) 1969 : «Les thèses de $1929 »$, Change 3, Paris, Seuil, 21-49.

Meschonnic Henri 1999 : Poétique du traduire, Lagrasse, Verdier.

Moirand Sophie 2004: «Le texte et ses contextes», in J.-M. Adam et al. Texte et discours : catégories pour l'analyse, Dijon, Editions Universitaires de Dijon, 129-143.

Pêcheux Michel 1990 : L'inquiétude du discours, textes de M. Pêcheux choisis et présentés par Denise Maldidier, Éditions des Cendres.

Prandi Michele 2007 : «Les fondements méthodologiques d'une grammaire descriptive de l'italien », Langages 167, 70-84.

Riegel Martin 2006: «Cohérence textuelle et grammaire phrastique », in Cohérence et discours, Frédéric Calas (dir.), Paris, Presses de l'Université Paris Sorbonne, 53-64.

Saussure Ferdinand de 2002 : Écrits de linguistique générale, Paris, Gallimard.

Slakta Denis 1975 : «L'ordre du texte », Études de linguistique appliquée 19, Paris, Didier, 30-42.

— 1977 : «Introduction à la grammaire de texte », Actes de la session de linguistique de Bourg-Saint-Maurice 4-8 septembre 1977, Paris, Publications du Conseil scientifique de la Sorbonne Nouvelle-Paris III, 6-63.

— 1985 : «Grammaire de texte : synonymie et paraphrase », in Aspects de l'ambiguité et de la paraphrase dans les langues naturelles, Catherine Fuchs éd., Berne, Peter Lang, 123-140.

Soutet Olivier 2005 [1995] : Linguistique, Paris, PUF-Quadrige.

Viprey Jean-Marie 2006a : «Quelle place pour les sciences des textes dans 1'Analyse de Discours », Semen $n^{\circ} 21$, Besançon, PU de Franche-Comté, 167-182.

— 2006b : «Structure non-séquentielle des textes », Langages n 163, Paris, Larousse, 71-85.

Weinrich Harald 1973 (1964) : Le Temps, Paris, Seuil.

Wilmet Marc 1997 : Grammaire critique du français, Paris, Hachette-Duculot.

i Je reprends ici une partie d'une conférence donnée en qualité de professeur associé de Paris IV (semestre d'hiver 2007-2008) le 6 décembre 2007, dans le cadre des «Conférences de linguistique de la Sorbonne » organisées par Olivier Soutet.

ii J'aborde cette question de l'évolution de la position de Barthes dans Adam 2001b. 